\title{
Evolutionary origin of power-laws in Biochemical Reaction Network; embedding abundance distribution into topology
}

\author{
Chikara Furusawa ${ }^{1}$ and Kunihiko Kaneko ${ }^{2,3}$ \\ 1 Department of Bioinformatics Engineering, \\ Graduate School of Information Science and Technology, \\ Osaka University, 2-1 Yamadaoka, Suita, Osaka 565-0871, Japan \\ ${ }^{2}$ Department of Pure and Applied Sciences Univ. of Tokyo, \\ Komaba, Meguro-ku, Tokyo 153-8902, Japan \\ 3 ERATO Complex Systems Biology Project, JST, \\ 3-8-1 Komaba,Meguro-ku, Tokyo 153-8902, Japan
}

\begin{abstract}
The evolutionary origin of universal statistics in biochemical reaction network is studied, to explain the power-law distribution of reaction links and the power-law distributions of chemical abundances. Using cell models with catalytic reaction network, we find evidence that the powerlaw distribution in abundances of chemicals emerges by the selection of cells with higher growth speeds. Through the further evolution, this inhomogeneity in chemical abundances is shown to be embedded in the distribution of links, leading to the power-law distribution. These findings provide novel insights into the nature of network evolution in living cells.
\end{abstract}

PACS numbers: 87.17.Aa, 87.23.Kg, 89.75.Fb 
Recent advances in biology have provided detailed knowledge about individual molecular processes and their functions. Despite its enormous success, it is increasingly clear that the nature of intra-cellular dynamics maintaining the living state is difficult to be understood only by building up such detailed knowledge of molecules, since a complex network of reactions among these molecules, such as proteins, DNA, RNA and so forth, are essential for it. One possible strategy to extract the nature of intra-cellular dynamics is to search for universal laws with regard to the networks of intra-cellular reactions common to all living systems, and then to unravel the dynamics of evolution leading to such universal features.

Indeed, recent large-scale studies revealed two universal features in cellular dynamics. First, the power-law distribution of links in reaction networks was discovered in metabolic and other biochemical reaction networks, as is termed as a scale-free network, where the connectivity distribution $P(k)$ obeys the law $k^{-\gamma}$ with $\gamma \approx(2 \sim 3)[1,2$, 3, 4, 5]. Second, the abundances of chemicals in intra-cellular reaction were found to also exhibit the powerlaw distributions, as confirmed at the levels of gene expression [6, 7, 8] and metabolic flux 9]. Here, the chemical abundances plotted in the order of their magnitude are inversely proportional to their rank.

Despite the potential importance of these universal statistical laws, however, how they are formed through evolution and how the two laws are mutually related are still unknown. Here we answer these questions through analysis and simulations of evolution of a simple cell model, to demonstrate that a power-law distribution in abundances of chemicals emerges as a result of competition for higher growth of a cell, and then this abundance inhomogeneity is embedded into the distribution of links, leading to the scale-free network with hierarchical organization of reaction dynamics. The findings provide novel insights into the nature of network evolutions in living cells.

To determine the emergence and interrelationships of the power-laws in chemical abundances and network connectivity through the process of evolution, we adopted a simple model of intracellular reaction dynamics that captures the catalytic reaction processes essential for cell growth and division, following Ref. 6, 10, 11]. Although this model was chosen simply to satisfy the minimal requirements of intracellular reaction dynamics of a growing cell, it was found to capture universal statistical behaviors as confirmed experimentally [6]. By studying a class of simple models with these features and the evolution of the network of the reaction, we study how the power-laws in abundances and network connectivity emerge 
inevitably.

Consider a cell consisting of a variety of chemicals. The internal state of the cell can be represented by a set of concentrations $\left(x_{1}, x_{2}, \cdots, x_{K}\right)$, where $x_{i}$ is the intra-cellular concentration of the chemical species $i$ with $i$ ranging from $i=1$ to $K$. Depending on whether there is an enzymatic reaction from $i$ to $j$ catalysed by some other chemical $\ell$, the reaction path is connected as $(i+\ell \rightarrow j+\ell)$. The rate of increase of $x_{j}$ through this reaction is given by $x_{i} x_{\ell}$, where for simplicity all of the reaction coefficients were chosen to be 1 .

Next, some nutrients were supplied from the environment by transportation through the cell membrane with the aid of some other chemicals, i.e., "transporters". Here, we assumed that the transport activity of a chemical is proportional to its concentration, and the rate of increase of $x_{i}$ by the transportation is given by $D x_{m_{i}}\left(X_{i}-x_{i}\right)$, where $m_{i}$ is the transporter for the nutrient $i, D$ is a transport constant and the constant $X_{i}$ is the concentration of the $i$-th chemical in the environment. In addition, we took into account the changes in cell volume, which varies as a result of transportation of chemicals into the cell from the environment. For simplicity, we assumed that the volume is proportional to the sum of chemicals in the cell. The concentrations of chemicals are diluted due to increases in volume of the cell, which imposes the restriction $\sum_{i} x_{i}=$ const. When the volume of a cell is doubled due to nutrient intake, the cell is assumed to divide into two identical daughter cells.

To summarize these processes, the dynamics of chemical concentrations in each cell are represented as

$$
d x_{i} / d t=R_{i}-x_{i} \sum_{j} R_{j}
$$

with

$$
\begin{aligned}
R_{i}= & \sum_{\substack{j, \ell\\
}} \operatorname{Con}(j, i, \ell) x_{j} x_{\ell}-\sum_{j^{\prime}, \ell^{\prime}} \operatorname{Con}\left(i, j^{\prime}, \ell^{\prime}\right) x_{i} x_{\ell^{\prime}} \\
& \left(+D x_{m_{i}}\left(X_{i}-x_{i}\right)\right),
\end{aligned}
$$

where $\operatorname{Con}(i, j, \ell)$ is 1 if there is a reaction $i+\ell \rightarrow j+\ell$, and 0 otherwise, while the last term in $R_{i}$ is added only for the nutrients, and represents its transportation into a cell from the environment. The last term in $d x_{i} / d t$ with the sum of $R_{j}$ represents dilution effect due to changes in cell volume.

Of course, how these reactions progress depends on the intra-cellular reaction network. Here, we study the evolution of the network, by generating slightly modified networks and 
selecting those that grow faster. First, $n$ mother cells are generated, where the connection paths of catalytic network were chosen randomly such that the number of incoming, outgoing, and catalyzing paths of each chemical is set to the initial path number $k_{\text {init }}$. From each of $n$ mother cells, $m$ mutant cells were generated by random addition of one reaction path to the reaction network of the parent cell. Then, reaction dynamics were simulated for each of the $n \times m$ cells to determine the growth speed of each cell, i.e., the inverse of the time required for division. Within the cell population, $n$ cells with faster growth speeds were selected as the mother cells of the next generation, from which $m$ mutant cells were again generated in the same manner.

A number of network evolution simulations were performed using several different initial networks, different parameters and various settings. We found that all of the simulations indicated common statistical properties with regard to both reaction dynamics and topology of networks. Here, we present an example of simulation results to show the common properties of our simulations.

The rank-ordered concentration distributions of chemical species in several generations are plotted in Fig.1, in which the ordinate indicates the concentration of chemical species $x_{i}$ and the abscissa shows the rank determined by $x_{i}$. The slope of the rank-ordered concentration distribution increased with generation, and within a few generations converged to a power-law distribution with an exponent -1, which was maintained over further generations. Or equivalently, the distribution $p(x)$ of the species with abundance $x$ is proportional to $x^{-2}$ [12].

The emergence of such a power-law by selection of cells with faster growth speeds is a natural consequence of our previous observations. To increase the cellular growth speed, changes in the network that enhance nutrient uptake from the environment are favored. This nutrient uptake is achieved by increasing the concentrations of transporters. However, the cell will no longer be able to grow continuously if there is excessive nutrient uptake as there will be insufficient room to synthesize the catalysts required to "metabolize" the nutrients and transporters. Thus, there is a critical value of nutrient uptake at which the cell reaches the maximal growth speed. At this point, the power-law distribution of chemical abundance appears in the intracellular dynamics, where the hierarchy of the successive catalytic reaction processes is organized [6]. With the evolutionary process shown in Fig.1, the nutrient uptake increases to accelerate the cellular growth speed until further mutations 
in the network result in exceeding the above critical value of nutrient uptake. Here, successive increases in growth speed by "mutation" to the reaction network are possible only when the degree of enhancement of nutrient uptake by the mutation is synchronized with increases in other catalytic activities. Consequently, selection favors networks in which nutrient uptake is maintained close to this critical point, where successive catalytic reaction processes maximize the use of nutrients and form a power-law distribution of chemical abundances.

Next, we investigated the topological properties of the reaction networks. Although both initial network generation and reaction path addition were random, after this evolutionary process the topological properties of networks deviated significantly from those expected from random networks. The connectivity distributions $P(k)$ of chemical species obtained from the network of the 1000th generation are plotted in Fig.2a, where $k_{\text {in }}, k_{\text {out }}$ and $k_{\text {cat }}$ indicate the numbers of incoming, outgoing and catalyzing paths of chemicals, respectively. These distributions were fitted by power-laws with an exponent close to -3. Thus, a scale-free network was approached through evolution, while this power-law behavior was maintained for further evolutionary processes.

As shown in Fig.3, in this simple model, the evolved reaction network formed a cascade structure in which each chemical species was mainly synthesized from more abundant species. That is, almost no chemical species disrupted the flow of chemical reaction from the nutrients, as the network approached that with optimal cell growth. It should also be noted that the reaction dynamics for each chemical were also inhomogeneous in that synthesis of each chemical species had a dominant reaction path. Such uneven use of local reaction paths was also reported previously in real metabolic networks [9].

The emergence of the scale-free-type connectivity distribution by this evolutionary process can be explained by selection of preferential attachment of paths to more abundant chemical species. Note that the power-law distribution of chemical abundance has already been established through evolution. Here, the connection of a reaction path to a more abundant chemical is more effective resulting in a change in growth speed of a cell. For example, assume that the change in growth speed by addition of an outgoing path from a chemical increases linearly with its abundance $x$. This assumption is natural as the degree of influence on the cellular state is generally proportional to the flux of the reaction path added to the network, i.e., the product of substrate and catalyst abundances. In this simple case, the probability $q_{\text {out }}(x)$ of having such an outgoing path after selection will increase linearly with $x$ even 
though the change in the network is random. Here, the connectivity distribution $P\left(k_{\text {out }}\right)$ is obtained by the transformation of variable as follows. Suppose that the probability of selection of a path attached to a chemical with abundance $x$ is given by $q(x)$, then the path number $k \propto q(x)$. By the transformation $k=q(x)$, the distribution

$$
P(k)=d x / d k p(x)=q^{\prime}\left(q^{-1}(k)\right) p\left(q^{-1}(k)\right)
$$

is obtained. By applying the abundance power-law $p(x) \propto x^{-2}$, we obtain $P(k)=k^{-(\alpha+1) / \alpha}$ when $q(x)=x^{\alpha}$. Consequently, a scale-free network with exponent -2 should be evolved if $q_{\text {out }}(x) \propto x$.

Numerically, we found that the probabilities $q_{\text {out }}(x)$ and $q_{\text {cat }}(x)$ were fitted by $q(x) \propto x^{\alpha}$ with $\alpha \approx 1 / 2$, as shown in Fig.2b. Then, using the above transformation the connectivity distribution was obtained as $P(k)=k^{-3}$. Here, it is interesting to note that the connectivity distribution observed from real metabolic and other biochemical networks follow the powerlaw $P(k) \propto k^{-\gamma}$ with $\gamma$ between 2 and 3, as often seen in experimental data [1, 2].

The probability $q(x)$ is determined through the evolutionary process. To clarify the reason for $q(x) \sim x^{\alpha}$ with $\alpha<1$ in outgoing and catalyzing paths, we investigated the relationship between substrate abundance $x$ and catalyst abundance $y$ of a path to be selected. For this, we simulated changes in growth speeds by random addition of a reaction path to the network of 200th generation. By examining only the mutants of $0.05 \%$ of the highest growth speeds we found that a path with small flux is not selected since adding such path cannot change the cellular state enough, while a path with large flux is not selected also, since such large change destroys hierarchical structure of catalytic reactions, which results the decrease of nutrient intakes or exceeding the critical point so that the "cell" can no longer grow. Then by plotting the substrate abundance $x$ and catalyst abundance $y$ for the selected paths on the $x-y$ plane, we found that the fluxes of the selected paths satisfy $\Delta<x y<\Delta+\delta$, with $\Delta=3.8 \times 10^{-8}$ and $\delta=4.0 \times 10^{-6}$ (data not shown). We also found that the density of paths to be selected is almost constant in the above region. Consequently, for each chemical $x$, the probability that such a path exists is given by the probability that there is such a partner chemical with abundance $y$, which satisfies $\Delta / x<y<(\Delta+\delta) / x$. That is,

$$
q(x)=\int_{\Delta / x}^{(\Delta+\delta) / x} p(z) d z \approx p(\Delta / x)(\delta / x)
$$


By using the equation (1), we obtain

$$
P(k)=\frac{-p(\Delta / y)}{(p(y)+y d p(y) / d y)) y^{2}},
$$

with $y p(y)=k$. Indeed, if $p(x)=x^{-2}$, the above expressions lead to $q(x) \propto x$, as well as $P(k)=k^{-2}$. This expression holds when the evolved network is just at the critical point. The evolved network is near this critical point but there is a slight deviation, as can be seen in the deviation from the power-law in Fig.1, for small abundance of chemicals. Note that the asymptotic behavior for large $k$ is given for small $y$. Then, the asymptotic behavior for large $k$ is given by $P(k) \approx 1 /((p(y)+y d p(y) / d y))$ depends on $p(y)$ for small $y$. If the asymptotic behavior of $p(y)$ for small $y$ is given by $y^{-\beta}$ with $\beta<2$, then $P(k) \approx k^{\beta /(1-\beta)}$. As $\beta<2$, the exponent of the power is smaller than -2 . For example, for $\beta=3 / 2$ (which corresponds to the relationship between $x$ and rank $n$ as $x \sim n^{-2}$ for large $n$, as seen in Fig.1), $P(k) \approx k^{-3}$ is obtained. In general, even if the behavior of $p(y)$ for small $y$ is not fitted by a power-law, its increase with $y \rightarrow 0$ is slower than $y^{-2}$. Then the decrease of $P(k)$ with $k$ is faster than $k^{-2}$, as often seen in experimental data [1, 2].

On the other hand, as $k_{i n}$ increases, the corresponding chemical has greater flow such that the correlation between the abundance $x$ and $k_{i n}$ was formed as in Fig.2c, leading to the scale-free network as shown in Fig.2a.

With regard to evolution of reaction networks, preferential attachment to a more connected node has often been discussed [1, 13]. In contrast, in the present mechanism the attachment is not preferential at all. Furthermore, it is different in two important respects. First, the dynamics of chemical abundance in the networks were introduced explicitly (described as node 'strength' in [14]), while previous models generally considered only the topological structure of the network. Second, selection only by cellular growth speed results in such a preference, even though attachment itself is random. Here, we found that more abundant chemical species acquired more reaction links as attachments of new links to such chemicals have both a greater influence on the cellular state and a higher probability of being selected. With these mechanisms, the power-law in abundance is naturally embedded in the intracellular reaction network structure through evolution, which is simply a process of selecting cells with faster growth speeds.

The emergence of two statistical features here is quite general, and does not rely on the details of our model, such as the kinetic rules of the reactions or the parameters used. In- 
stead, it is a universal property of evolution of intracellular reaction dynamics and networks, selected by growth speed in a fixed environment. The power-laws of both abundance and connectivity, which are often observed in intracellular reactions, are simply consequences of our mechanism by Darwinian selection.

We would like to thank T. Yomo and K. Sato for stimulating discussions. The work is supported by Grant-in-Aids for Scientific Research from the Ministry of Education, Science and Culture of Japan.

[1] Jeong, H. et al. Nature 407 (2000), 651

[2] Jeong, H., et al. Nature 411 (2001), 41

[3] Li, S. et al. Science 303 (2004), 540

[4] Featherstone, D. E. et al. Bioessays 24 (2002) 267

[5] Guelzim, N. et al. Nature Genet. 31 (2002), 60

[6] Furusawa, C., and Kaneko, K. Phys. Rev. Lett. 90 (2003) 088102

[7] Ueda, H. R. et al. Proc. Natl Acad. Sci. USA 101 (2003), 3765

[8] Kuznetsov, V. A. et al. Genetics 161 (2002) 1321

[9] Almaas, E. et al. Nature 427 (2004) 839

[10] Kaneko, K., and Yomo, T. Jour. Theor. Biol. 199 (1999) 243

[11] Furusawa, C., and Kaneko, K. Phys. Rev. Lett. 84 (2000), 6130

[12] The rank distribution, i.e., the abundances $x$ plotted by rank $n$ can be transformed to the density distribution $p(x)$, the probability that the abundance is between $x$ and $x+d x$. Since $d x=d x / d n \times d n$, there are $|d x / d n|^{-1}$ chemical species between $x$ and $x+d x$. Thus, if the abundance-rank relation is given by a power-law with exponent $-1, p(x)=|d x / d n|^{-1} \propto n^{2} \propto$ $x^{-2}$.

[13] Barabási, A.-L., and Albert, R. Science 286 (1999) 509

[14] Barrat, A., Barthélemy, M., and Vespignani, A. Phys. Rev. Lett. 92 (2004), 228701 


\section{Figure Captions:}

\section{Figure 1:}

Rank-ordered concentration distributions of chemical species. Distributions with several different generations are superimposed using different colors. The solid line indicates the power-law $x \propto n^{-1}$ for the reference. This power-law of chemical abundance is established around the 10th generation, and is sustained for further evolutions in the network. In the simulation, the growth speeds of $10 \times 2000$ networks were measured, and the top 10 networks with regards to the growth speed were chosen for the next generation. The parameters were set as $K=1000, D=4.0$, and $k_{\text {init }}=4$.

\section{Figure 2:}

Evolution of the network topology. (a), Connectivity distribution $P(k)$ of chemical species obtained from the network of the 1000th generation. The solid line indicates the power-law $P(k) \propto k^{-3}$. For comparison, the distribution of $k_{\text {rand }}$, obtained by a randomly generated reaction network with the same number of paths with the network of 1000th generation, is shown. (b), Probability $q(x)$ that a path to a chemical with abundance $x$ is selected in evolution. The probabilities for incoming $\left(q_{i n}(x)\right)$, outgoing $\left(q_{\text {out }}(x)\right)$, and catalyzing paths $\left(q_{\text {cat }}(x)\right)$ are plotted. The data were obtained by $1.5 \times 10^{5}$ trials of randomly adding a reaction path to the network of the 200th generation, and the paths giving the top $0.05 \%$ growth speeds were selected.

\section{Figure 3:}

Changes in the network structure. The abscissa shows the rank determined by the abundance of substrate $i$, and the ordinate shows the rank for the product $j$ : the top left is the most abundant and the bottom right is the least abundant. A point is plotted when there is a reaction path $i \rightarrow j$, while the abundance of catalyst for the reactions is given by different colors determined by rank. As each product is dominantly synthesized from one of the possible paths, we plotted only the path with the highest flow. (a), The network at the 10th generation, where the network structure is rather random, even though the power-law in abundance has already been established. (b), The network at the 1000th generation. Only a small number of paths are located in the upper-right triangular portion of the figure, indicating that almost all chemical species were synthesized from more abundant species. 


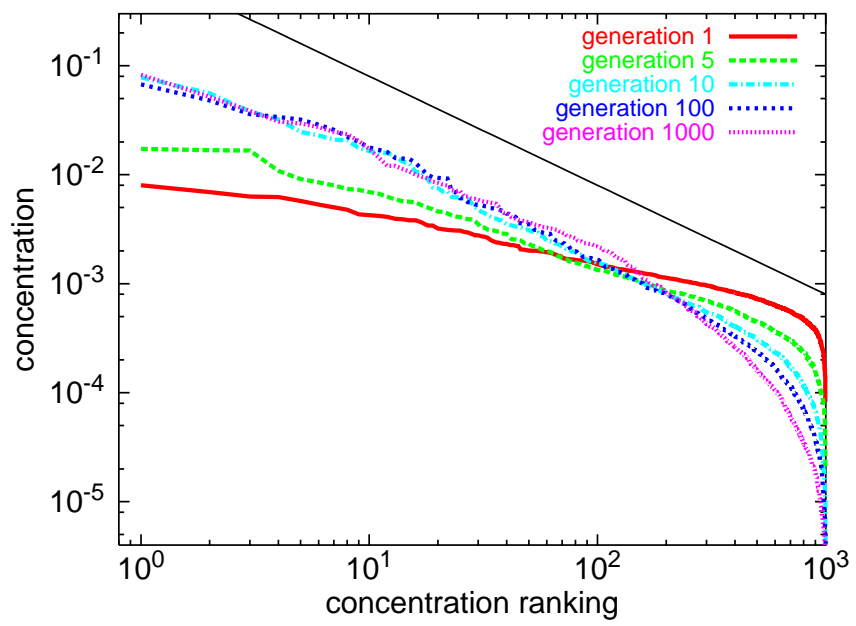

FIG. 1:

(a)

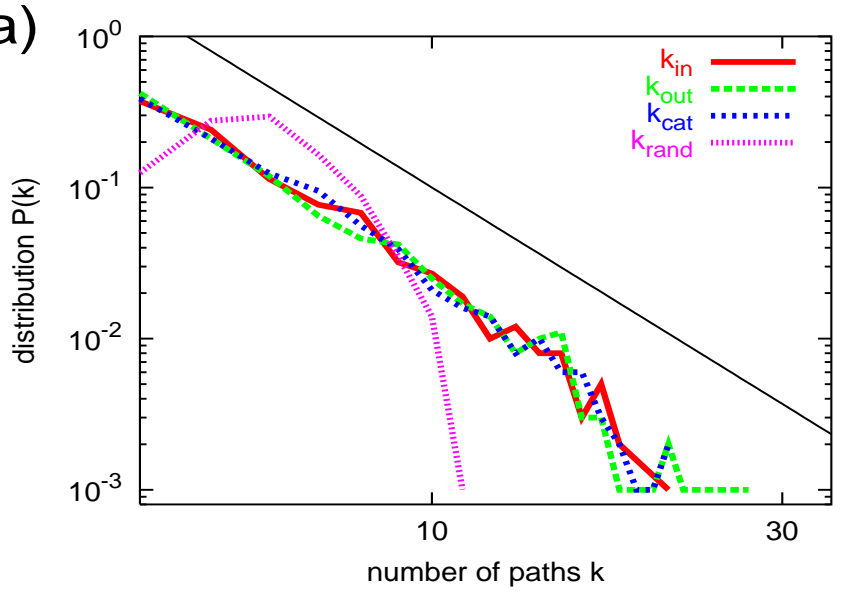

(b)

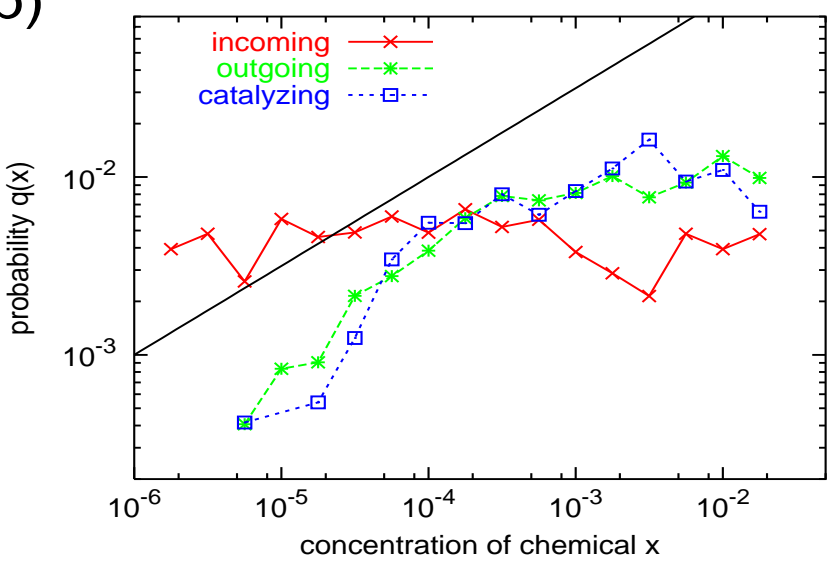

FIG. 2: 
(a)

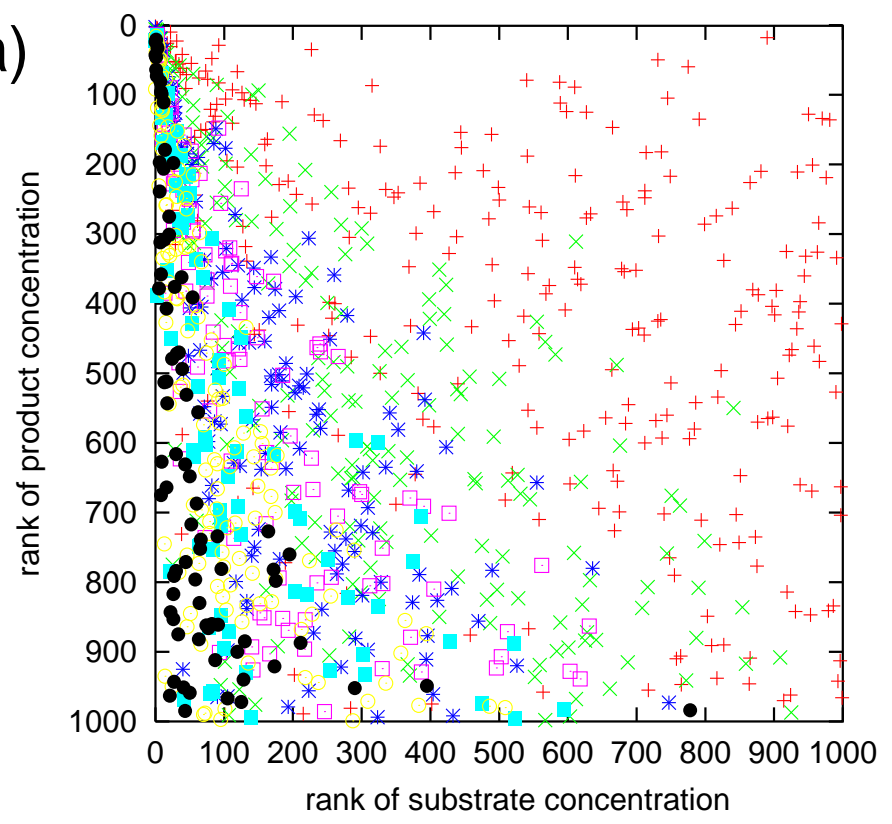

(b)

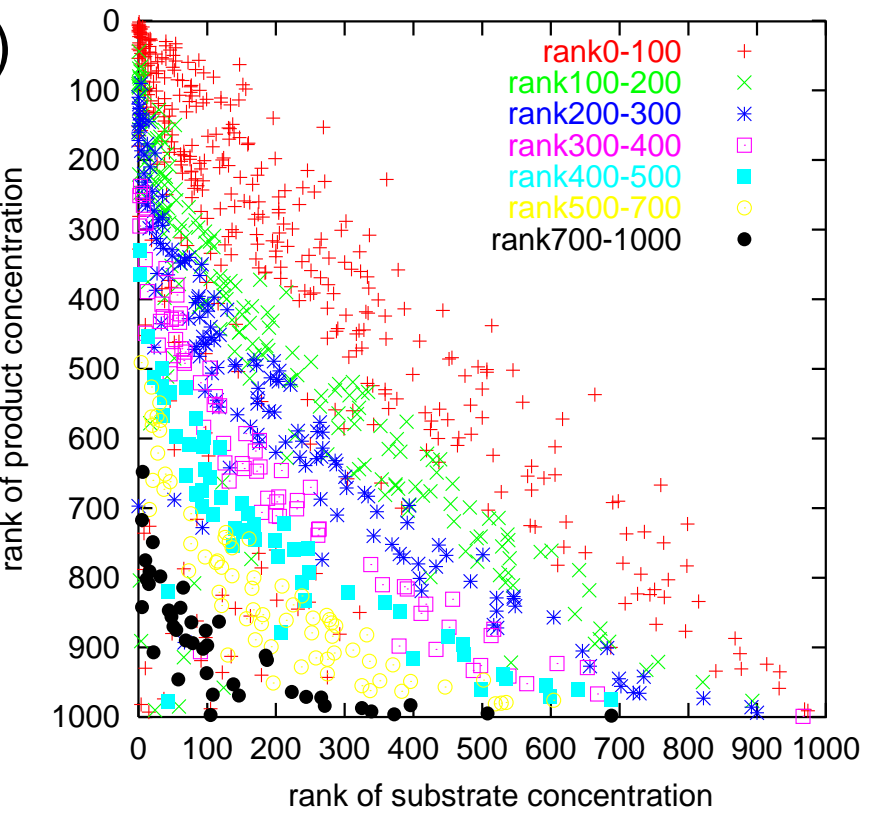

